\title{
ANÁLISE DE UMA EXPERIÊNCIA DE SUPERVISÃO CLÍNICA ${ }^{1}$
}

\author{
Helena Bazanelli PREBIANCHI ${ }^{2}$ \\ Dr. Mauro Martins AMATUZZI ${ }^{3}$
}

\begin{abstract}
RESUMO
Trata-se de umaanálise qualitativa da experiência de umgrupo de supervisão clínica, do curso de Psicologia da PUC-Campinas. Oinstrumento Versão de Sentido (VS) foi utilizado para estudar o processo de desenvolvimento do supervisando e a dinâmica da relação supervisor-supervisando, através da descrição da experiência do grupo em 7 encontros. Os resultados indicaram que houve estágios no desenvolvimento dos supervisandos e alterações correlatas no papel do supervisor, como proposto pelos modelos desenvolvimentistas de supervisãoe pelomodelo daAbordagem Centradana Pessoa. A flexibilidade do supervisore sua habilidade emavaliarasnecessidades dos supervisandos foram essenciais para o estabelecimento de uma relação produtiva de supervisão. As relações cliente-supervisando e gruposupervisando influenciaram o processo de supervisão. O estudo sugeriu também, que aidentidade profissional dos supervisandos nãosurgiuapenas como função da apreensão das técnicas psicoterápicas.
\end{abstract}

Palavras-chave: supervisão clínica, supervisando emodelos de supervisão

\begin{abstract}
It's a qualitative analysis of a clinical supervision group's experience from PUC-Campinas Psychology Course. Aninstrumentnamed Versãode Sentido (VS) was used to study the process of supervisee development and the dynamic of supenisor-supervisee relation through the description of the group members'experienceduring 7 meetings. The results indicated that there were stages in the supervisees' development and related alteracions in the supervisor's role. These findings were consistent with the developmental supervision models and the Person Centered Approach to supervision. The supervisor's flexibility and his ability to evaluate the supervisees'needs were essential toestablishaproductivesupenvision relationship. The client-supervisee relationand the group-supervisee relation influenced the supervision process. Finally, the studysuggested that the supervisee's professional identity wasnot only a function of the psychotherapy techniques knowledge.
\end{abstract}

Key words: clinical supervision, supervisees, supervision models

\footnotetext{
(1) Trabalho apresentado no II ENCONTRO SOBRE PSICOLOGIA CLÍNICA - MACKENZIE, maio de 1999. Endereço para Correspondência: Rua Vieira Bueno,184 -Cambuí - Campinas/SP - CEP 13024-040

(2) Mestre em Psicologia Clínica - Endereço para correspondência: Rua Marechal Deodoro, 1099 Centro - Campinas- SP CEP 13030-310

(3) Prof. Dr. PUC-Campinas Endereço para correspondência: Rua Marechal Deodoro, 1099 centro Campinas, SP.
} 
A aquisição do conhecimento teórico-formal e a supervisão clínica constituem-se nos pilares principais, sob os quais se assenta a formação do psicoterapeuta. A dimensão teórica espera-se seja adquirida formalmente dentro de um currículo que inclua teoria e pesquisa relacionadas à terapia, de forma a prover ao aluno os aspectos técnicos essenciais ao seu desempenho profissional.

Quanto a supervisão (normalmente realizada em grupos, durante o período de formação), pode-se pensar o seu objetivo último, como sendo o desenvolvimento de habilidades práticas e conceituais dos jovens terapeutas, por terapeutas experientes. De acordo com Bernard e Goodyear (1992), supervisão é uma intervenção fornecida por um membro senior de uma profissão, a um ou mais membros juniores da mesma profissão. E ainda: esta relação é avaliativa, se estende no tempo e tem os propósitos, simultâneos, de incremento do funcionamento profissional dos membros juniores, monitorando a qualidade dos serviços profissionais oferecidos aos clientes que eles atendem, servindo como porteiro para os que estão iniciando na profissão em questão (Bernard e Goodyear, 1992).

De modo geral, nos cursos de Psicologia, é no âmbito da supervisão que se inicia a formação da identidade profissional, pois é neste espaço que acontece a articulação entre o que o principiante conhece sobre a teoria, o seu cliente e o que experimenta na relação terapeutacliente.

Simultaneamente, a supervisão provê um elemento de socialização, pois o supervisor tem a profissão aspirada pelo supervisando e nesse sentido, pode servir como modelo.

Ainda que reconhecida como uma atividade central na formação do terapeuta, a supervisão, até há pouco tempo, não era distinguida com cuidados técnicos, com sistematização ou organização. Buys (1987) ao constatar a ausência de interesse e/ou estudos científicos nessa área, comenta: "A atividade de supervisão é intuitiva, decorre da prática em outra atividade, ou podemos até dizer, que a atividade de bem supervisionar vem como 'vale brinde' dentro da atividade psicoterapêutica" (p. 11-12).

Assim é, que por muito tempo a supervisão se processou dentro de um modelo psicoterápico. Isto é, a teoria psicoterápica adotada pelo supervisor era aplicada tanto ao contexto da terapia, quanto ao contexto da supervisão.

Se é verdade que esse modelo de supervisão tem a vantagem de maximizar a integração entre a teoria e o treinamento; por outro lado, tem implícito,o problema de que se o supervisor tratar o supervisando de forma similar ao cliente, este poderá sentir-se e comportar-se como cliente.

Mais recentemente, contudo, o processo de supervisão e suas variáveis, têm merecido maior interesse científico, originando inúmeras pesquisas a partir de meados da década de 80 . Novos modelos conceituais de supervisão têm sido propostos, concebendo-a como um processo à parte da terapia. Nesse sentido, os modelos desenvolvimentistas ${ }^{2}$, de supervisão, por sua natureza a teórica, podem ser usados por várias abordagens psicoterápicas.

Tais modelos partem do pressuposto de que há uma seqüência lógica dos estágios pelos quais os supervisandos devem passar no seu desenvolvimento e que esta é aproximadamente a mesma para qualquer supervisando. Os modelos desenvolvimentistas focalizam não só o desenvolvimento do supervisando, como também o do supervisor. De modo geral, os diversos modelos desenvolvimentistas têm seu foco central nos supervisandos e suas interações com o supervisor.

(2) Desenvolvimentista: ainda que de uso incomum na língua portuguesa, o termo é adotado por Lara Campos (1988, conforme bibliografia referida ao final desse artigo) para se referir aos modelos de supervisão baseados nas teorias sobre o desenvolvimento humano. 
No Brasil, são raríssimas as pesquisas sobre a supervisão. Num estudo realizado por Campos (1994) os resultados indicaram que os supervisores brasileiros não possuem qualquer treinamento formal ou informal para exercer sua atividade e que desconhecem qualquer avanço sobre a ciência da supervisão.

Como escreve Campos (1998): "A maioria dos supervisores iniciou sua prática baseada exclusivamente em sua própria experiência, em um momento em que a literatura específica era diminuta, restrita ou mesmo inexistente, de tal modo que a grande maioria dos profissionais, tende a utilizar sua própria experiência pessoal para conduzir a supervisão (p. 29).

Supervisora clínica, há doze anos, na Pontifícia Universidade Católica de Campinas, não fujo ao perfil traçado por Campos. Minha prática docente reflete muito mais a minha história de vida e meu percurso profissional do que um treinamento específico ou uma formação teórico/conceitual que permitam enquadrar ou realmente compreender o processo de supervisão. Desse modo, a possibilidade de realização deste estudo, pareceu-me afinada com meus objetivos de conhecer alguns aspectos do processo de supenvisão relacionado ao desenvolvimento dos supervisandos e à relação supervisor-supervisando.

Lembrando-se que na supervisão, o foco é o conteúdo da sessão realizada pelo supervisando, pode-se afirmar que o foco é o relato da relação supervisando-cliente. Sob esse prisma, parece acertado concluir que a supervisão é o lugar onde além das dúvidas teóricas e técnicas, emerge a necessidade de auto-conhecimento do supervisando.

Dentro de uma perspectiva Fenomenológico-Existencial, a descrição da experiência com o cliente possibilita ao supervisando entrar em contato consigo mesmo, com seus questionamentos e inseguranças (Moreira, 1997).

De maneira análoga, podemos supor que também a relação supervisor-supervisando, possa ser compreendida por ambos, a partir da descrição de suas experiências.

Ultimamente, um tipo de relato, denominado Versão de Sentido (VS), vem sendo utilizado como instrumento de formação e pesquisa qualitativa. Trata-se de um relato livre que "consiste numa fala expressiva da experiência imediata de seu autor, diante de um encontro recém-terminado" (Amatuzzi, 1993).

Enquanto produto, a VS é um texto expressivo da experiência imediata, escrito ou gravado por iniciativa da própria pessoa, ou solicitado por um interlocutor. Dessa forma, a leitura de uma VS pode ocorrer em dois níveis: como transmitindo a vivência de seu autor e também, o sentido da relação (Amatuzzi, 1993). Isto é, a versão de sentido, ao enfatizar as sensações do seu autor através da descrição do vivido, faz emergir a intersubjetividade da relação, transformando-a em algo com sentido.

O trabalho com séries de VS é, pode levar ao conhecimento do sentido de processos, e portanto, espera-se que o uso da VS como método aplicado a um grupo de supervisão, dê a conhecer alguns aspectos do processo de transformação do aluno em terapeuta e da relação supervisor-supervisando.

Nesse sentido, delineou-se o presente estudo, com o objetivo geral de conhecer alguns aspectos do processo de supervisão e de modo específico, conhecer aspectos do desenvolvimento das supervisandas e da relação supervisor-supervisanda.

\section{MÉTODO}

\section{SUJEITOS:}

Os sujeitos envolvidos neste trabalho foram oito alunas e a supervisora que compõem um dos grupos de Estágio Supervisionado em Psicologia Clínica, do 5ㅇa ano do curso de Formação de Psicólogos da Pontifícia Universidade Católica de Campinas. Trata-se de uma 
disciplina anual e obrigatória, dentro da qual, cada uma das alunas (estagiárias) foi responsável pelo atendimento clínico de um paciente, na Clínica de Psicologia do Instituto de Psicologia daPUC-Campinas.

No caso específico desse grupo de estagiárias, os atendimentos referiram-se a sete pacientes adultos do sexo feminino e a um adolescente do sexo masculino. Os encontros com os pacientes ocorreram semanalmente, em sessões com 50 minutos de duração. As supervisões também foram semanais e tiveram duração de 5 horas/aula.

O presente trabalho teve início na $4^{a}$ supervisão, estendendo-se até o último encontro do $1^{\circ}$ semestre de 1998 , num total de 7 encontros.

\section{ESTRATÉGIA:}

Foi solicitado às supervisandas, que ao final da supervisão, escrevessem de forma suscinta, qual tinha sido o sentido do encontro.

À supervisora, coube a mesma tarefa.

\section{ANÁLISE DOS RESULTADOS:}

Os resultados foram analisados em quatro etapas sucessivas: a partir da leitura de todos os textos de todos os encontros (1), foi feito o levantamento dos temas comuns a cada um deles (2) e procedeu-se à sua tabulação (3). Finalmente, foi realizada a comparação entre os temas comuns intra-encontros e inter-encontros (4).

\section{RESULTADOS}

A Tabela 1 mostra os temas comuns das VSs. das supervisandas e o sentido presente nas anotações da supervisora,em cada um dos encontros realizados.
Analisados sob o aspecto inter-encontros, os textos das supervisandas parecem refletir a ocorrência de um processo, com etapas que se sucedem a partir de um sentimento de insegurança e vulnerabilidade frente ao cliente (Encontro 1: $\mathrm{E}_{1}, \mathrm{E}_{2} ; \mathrm{E}_{3} ; \mathrm{E}_{4} ; \mathrm{E}_{5}$ e $\mathrm{E}_{6} ;$ Encontro 2: $E_{2} ; E_{3} ; E_{5}$ e $E_{8}$ ) até um sentimento de autoconfiança e tranqüilidade (Encontro 5: $E_{1} ; E_{3} ; E_{5}$; $\mathrm{E}_{6}$; Encontro 6: $\mathrm{E}_{5} ; \mathrm{E}_{6}$; Encontro 7: $\mathrm{E}_{1} ; \mathrm{E}_{5} ; \mathrm{E}_{7}$ ). Esse percurso foi intermediado por encontros em que o sentido foi a distinção das individualidades (Encontro 3: $E_{1} ; E_{2} ; E_{3} ; E_{4} ; E_{5} ;$ Encontro 4: $E_{2} ; E_{3}$ e $E_{4}$ ).

Pode-se observar também, que no início do processo, as supervisandas almejaram o recurso técnico (Encontro 1: $E_{3} ; E_{6}$ e $E^{7}$; Encontro 2: $E_{4}$ ),para numa segunda etapa, buscarem respostas na Ética e em si próprias (Encontro 3: $\mathrm{E}_{1} ; \mathrm{E}_{2} ; \mathrm{E}_{3} ; \mathrm{E}_{4} ; \mathrm{E}_{5}$ e $\mathrm{E}_{6}$; Encontro 4: $\mathrm{E}_{3}$ e $\mathrm{E}_{4}$ ) e finalmente, as angústias foram respondidas na relação com o cliente e com o grupo (Encontro 5: $\mathrm{E}_{4} ; \mathrm{E}_{5}$ e $\mathrm{E}_{6}$; Encontro 6: $\mathrm{E}_{1} ; \mathrm{E}_{2} ; \mathrm{E}_{3} ; \mathrm{E}_{4}$ e $\mathrm{E}_{7}$; Encontro 7: $\mathrm{E}_{1} ; \mathrm{E}_{2} ; \mathrm{E}_{3} ; \mathrm{E}_{5} ; \mathrm{E}_{7}$ e $\mathrm{E}_{8}$ ).

Ainda analisando as VSs. das supervisandas, pode-se perceber que nos primeiros Encontros ( 1 e 2), o foco esteve nas necessidades individuais (isto é, falaram de preocupações individuais e referiram-se a um único cliente - o seu). No decorrer do processo, trataram de forma genérica do papel do terapeuta (Encontros 3 e 4), sendo apenas, ao final (Encontros 5,6 e7) que aconteceu o auto-reconhecimento como membro de um grupo com identidade profissional.

Paralelamente, os relatos da supervisora parecem traduzir a transformação de um sentimento inicial de impaciência e irritação (Encontros 1 e 2) em um sentimento de satisfação e entusiasmo (Encontros 5, 6 e 7). Unindo esses dois momentos, percebe-se uma fase intermediária, caracterizada por uma expectativa positiva (Encontros 3 e 4). 


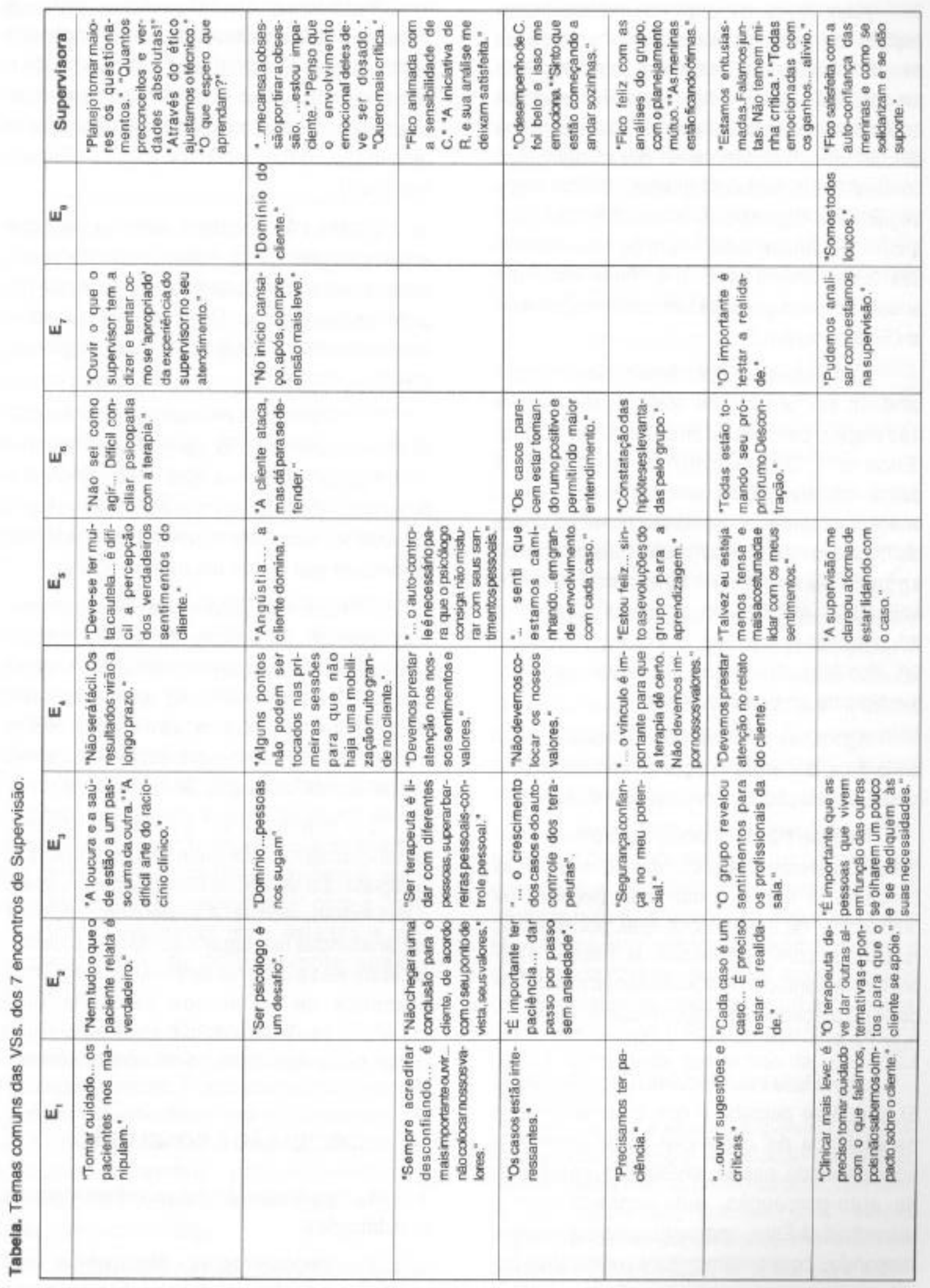

Rev. Estudos de Psicologia, PUC-Campinas, v. 17, n. 1, p. 55-63, janeiro/abril 2000 
Além dessa mudança no sentido, as anotações da supervisora quando analisadas seqüencialmente, relataram, a princípio, uma preocupação com a falta de profundidade dos questionamentos das supervisandas e com sua dificuldade em distanciar-se dos clientes (Encontros 1 e 2, respectivamente). Numa etapa seguinte, a capacidade e sensibilidade para análise e planejamento foram os seus interesses (Encontros 3, 4 e 5) e, finalmente, um anseio de que o grupo se tornasse independente (Encontros 6 e 7 ).

A alteração do papel da supervisora, pode também ser constatada como passando por três etapas: partindo de uma função de ensinar (Encontro 1:"O que espero que aprendam?") e definir objetivos (Encontro 2:"Penso que o envolvimento emocional delas deve ser dosado.", "Quero mais crítica"); para 2 - um papel de agente incentivador (Encontro 3:"Fico animada me deixam satisfeita"; Encontro 4:"...foi belo e isso me emociona", Encontro 5:"...fico feliz...") e então, 3 - se tornando um membro do grupo (Encontro 6).

Agora, se analisarmos os relatos intra encontros, podemos chegar ao sentido do processo da relação supervisora-supervisandas:

$\mathrm{Na}$ fase inicial, quando sentiram-se inseguras e vulneráveis aos clientes, as estagiárias buscaram o quê e como fazer (técnica) na "sabedoria" da supervisora, que, por sua vez, respondeu com sentimentos de irritação e impaciência e então procurou aumentar a profundidade da percepção das supervisandas (Encontros 1 e 2).

Já, na fase intermediária (Encontros 3,4 e 5), o quê se percebe é que o sentimento de necessidade de diferenciar-se do cliente foi acompanhado, nas supervisandas, pela busca da auto-percepção, auto-conhecimento e recorrência à Ética, enquanto que a supervisora respondeu com sentimento de expectativa positiva e incentivou a compreensão sensível e o planejamento da relação terapêutica.
Finalmente, na última fase, para as supervisandas, o sentimento de auto-confiança e identidade profissional, ocorreram com a identificação com o grupo e na relação terapêutica, sendo que nesse momento, a supervisora respondeu com entusiasmo e alívio (Encontros 6 e 7 ).

Porém, não podemos nos esquecer que esses movimentos da relação supervisora-estagiária, ocorreram a partir de e para a relação cliente-supervisanda. Dessa forma, a análise intra-encontros permite ainda as seguintes constatações:

1 - Num primeiro momento, a relação cliente-supervisanda foi percebida como ameaçadora, por esta última. Não havia possibilidade de um distanciamento do cliente e a relação supervisora-supervisanda foi caracterizada pela autoridade que a primeira representava.

2 - No meio do percurso, a relação clientesupervisanda, foi tratada como uma relação técnica. O distanciamento entre supervisanda e cliente foi maior; não havia, ou havia muito pouco de pessoal, na atuação da última. Nesse ponto, a relação supenvisora-supenvisanda teve um caráter de facilitação da experiência.

3 - Finalmente, a relação clientesupervisanda foi distinguida pela compreensão completa que se tem do cliente e pelo respeito à sua individualidade. A ansiedade inicial das supervisandas deu lugar a aceitação; a distância entre elas e o cliente diminuiu, em função da presença de elementos pessoais (das supervisandas). A relação com a supervisora então, foi de igualdade, ou não-dependência.

\section{DISCUSSÃO E CONCLUSÃO}

Os resultados obtidos permitem algumas constatações:

1 - Inegavelmente, observa-se uma seqüencialização no desenvolvimento das supervisandas. No caso específico desse 
grupo, os achados tanto falam similarmente aos modelos desenvolvimentistas de supervisão (por exemplo: Loganbill, 1982; Stoltenberg e Delworth, 1988) como ao modelo da Abordagem Centrada na Pessoa (Buys, 1987).

É possível reconhecer no grupo estudado, estágios sucessivos de confusão e integração em relação aos aspectos: consciência emocional, autonomia, identidade, respeito pelas diferenças individuais, propósito e direção ética (como proposto por Loganbill, 1982, ou como apontado por Stoltenberg e Delworth, 1988) notar três estruturas que dimensionam o desenvolvimento desses e outros aspectos: $1>$ a quantidade de dependência do supervisionado, 2> a auto-consciência e a consciência dos outros e $3>$ a motivação para 0 processo de desenvolvimento.

Paralelamente, os resultados podem também ser traduzidos pelas fases reconhecidas na supervisão da Abordagem Centrada na Pessoa: na primeira, há a apropriação da técnica pelo terapeuta e o uso da teoria na compreensão e explicação dos fenômenos ("como fazer'; "como entender"); na segunda fase, ocorre a adaptação à técnica através da acomodação afetiva e a significação experiencial da teoria e, por último, na terceira fase, acontece a apropriação da "atitude terapêutica" com a compreensão experiencial mais extensa e profunda do cliente pelo psicoterapeuta (Buys, 1987).

2 - O papel da supervisora se altera, de acordo com as transformações que se suscedem nas supervisandas. Como apontado nos modelos desenvolvimentistas referidos anteriormente, a princípio ela é a autoridade, da qual as iniciantes dependem; posteriormente é a catalisadora e suporte das mesmas e ao final, é vista como uma colega.

A supervisão na Abordagem Centrada na Pessoa, prevê também a mudança na atuação do supervisor: na primeira fase do processo, o supervisor faz intervenções didáticas técnicas centradas no psicoterapeuta e intervenções didáticas técnicas centradas no cliente; na fase seguinte, ele faz intervenções experienciais centradas no psicoterapeuta e intervenções experienciais centradas no cliente visando dar significado experiencial à teoria e na última fase, predominam as intervenções experienciais centradas no cliente, visando a compreensão experiencial dele, num nível mais profundo pelo terapeuta (Buys, 1987).

3 - Uma vez que há transformações na supervisanda e transformações na supervisora, parece-nos legítimo pensar, que a relação supenvisora-supervisanda é crucial ao desenvolvimento do processo de supervisão.

Como Campos (1998) salientou:"as distorções na relação entre o supervisor e o supervisionado são, muitas vezes, responsáveis pelo fracasso da supervisão" (p.85).

Nesse sentido, se tivermos como referência um modelo desenvolvimentista de supervisão, podemos concluir que a flexibilidade do supervisor e a sua capacidade de avaliação das necessidades do supervisando são elementos fundamentais para o estabelecimento de uma relação profícua.

Já, numa perspectiva humanista, a relação supervisor-supervisando, é o principal veículo para o desenvolvimento do terapeuta iniciante pois é na relação da supervisão, que ele reflete (com o supervisor) teórica, técnica e experiencialmente, sobre si próprio como psicoterapeuta na relação com o seu cliente. Como afirma Buys (1987): "a supervisão é uma prática intensiva da apreensão da relação na relação" (p.18).

4 - Sendo a relação supervisor-supervisando condição para apreensão da relação cliente-supervisando, podemos dizer, que existe, na supervisão, também uma relação supervisor-cliente-supervisando. Provavelmente, essa tríplice relação, contribui para o delineamento da atuação do supervisor (os resultados encontrados sobre como as alterações na 
relação cliente-supervisando são contingentes as variações na relação supervisor-supervisionado, parecem apontar nessa direção).

5 - Quando falamos de supervisão em grupo, somos obrigados a pensar na existência de outra relação na relação. Isto é: a relação grupo-supervisando. Por conseguinte, outra tríplice relação se torna possível: supervisor-grupo-supervisando. Considerando, que estas relações também se evidenciaram como muito importantes na formação do terapeuta (os resultados desse estudo, mostram que a identidade profissional surgiu no e com o grupo), é possível imaginar que assim como ocorre com o supervisor, o grupo pode funcionar facilitando ou restringindo o processo.

A despeito de a maior parte da bibliografia consultada, reconhecer a importância de o supervisor possuir habilidades específicas para condução de grupos, chama a atenção a quase que ausência de pesquisas sobre $o$ assunto, no aspecto que aqui abordamos.

6 - Os resultados evidenciaram, que, na realidade, a capacitação técnica é apenas, a fase inicial do desenvolvimento dos jovens terapeutas. De fato, a análise das VSs. das supervisandas, faz transparecer que a identidade profissional não surge como função direta da apreensão da técnica.

É provável, que tais resultados pudessem ser melhor compreendidos à luz de critérios mais definidos para a avaliação do que é chamado de "competência do terapeuta" ou "competência clínica". Ou, dialeticamente, esses resultados podem apontar em direção à definição.

As evidências encontradas de um processo de desenvolvimento do supervisando em direção a um terapeuta competente e da importância do supervisor nesse processo, reafirmam a necessidade de que a atuação desse profissional não seja reflexo de uma aquisição de conhecimento, exclusivamente empírica.
Sem um treinamento formal ou informal para o exercício da sua atividade, o supervisor encontrará dificuldades na clarificação de seu modelo de atuação e por conseguinte, a corrigibilidade será prejudicada.

Num país, em que a pesquisa sobre supervisão é ainda incipiente, o presente estudo sugere a possibilidade de alguma sistematização e organização para abordar esse processo. Reconhecemos que há muito por ser feito, ainda, nesse sentido, por isso esperamos que os supervisores brasileiros, sintam-se como nós, incentivados pela crença de que na ausência de critérios definidos não há como aperfeiçoar a supervisão, e em última análise, aperfeiçoar o psicoterapeuta.

\section{REFERÊNCIAS BIBLIOGRÁFICAS}

AMATUZZI, M.M. (1993) - O uso da versão de sentido na formação e pesquisa em psicologia, Coletâneas da ANPEPP, 11-24.

BERNARD, J.M. \& GOODYEAR, R.K. (1992) - Fundamentais of Clínical Supervision, Allyn \& Bacon, Boston.

BUYS, R.C. (1987) - Supervisão de Psicoterapia na abordagem humanista centrada na pessoa, Summus Editorial, São Paulo.

CAMPOS, LF.L (1998) - Formação, Supervisão e Treinamento em Psicologia Clínica, EP.U., São Paulo.

FRIEDLANDER, M.L. \& WARD, L.G. (1984) - Development and Valídation of Ihe Supervisory Styles Inventory, Journal of Connseling Psichology, 31(4). 541-557.

HOLLOWAY, E.L. (1988) - Models of Counselor Development or Training Models for Supervision? Rejoinder to 
Stoltemberg e Delworth, Professional Psychology: Research and Pratice, 19(2); 138-140.

MOREIRA, V. (1997) - Supervision en Psicoterapia: Un Enfoque Fenomenológico Existencial, Revista Terapia Psi- cológica, Ano XV, Volume VI (4), no 28, 93-99.

NEWMAN, A.S. (1981) - Ethical in the Supervision of Psychotherapy, Professional Psychology Reserch and Practice, 12(6): 690-695. 